\title{
Transverse Mode Revival of a Light-Compensated Quantum Memory
}

\author{
Fan Yang ${ }^{1}$, Torsten Mandel ${ }^{1}$, Christian Lutz ${ }^{1}$, Zhen-Sheng Yuan ${ }^{1,2}$, and Jian-Wei Pan ${ }^{1,2}$ \\ ${ }^{1}$ Physikalisches Institut, Ruprecht-Karls-Universität Heidelberg, \\ Philosophenweg 12, 69120 Heidelberg, Germany and \\ ${ }^{2}$ Hefei National Laboratory for Physical Sciences at Microscale and Department of Modern Physics, \\ University of Science and Technology of China, Hefei, Anhui 230026, China
}

(Dated: October 26, 2018)

\begin{abstract}
A long-lived quantum memory was developed based on light-compensated cold ${ }^{87} \mathrm{Rb}$ atoms in a dipole trap. The lifetime of the quantum memory was improved by 40 folds, from $0.67 \mathrm{~ms}$ to 28 ms with the help of a compensation laser beam. Oscillations of the memory efficiency due to the transverse mode breathing of the singly-excited spin wave have been clearly observed and clarified with a Monte-Carlo simulation procedure. With detailed analysis of the decoherence processes of the spin wave in cold atomic ensembles, this experiment provides a benchmark for the further development of high-quality quantum memories.
\end{abstract}

PACS numbers: 03.67.Hk, 32.80.Qk, 42.50.Gy

Quantum information processing (QIP) has promising advantages over classical information processing from the aspects of security, channel capacity and computing efficiency [1-4]. Therefore, one may expect that a quantum computer could solve a physical problem, say exploring the property of some new functional material, within a few seconds for which it takes several years with a classical computer. However, such a quantum computer has not yet been developed since the current QIP systems are not scalable which means the required resources increase exponentially along the complexity of the system [5]. This situation is going to be changed by integrating the quantum memory, a storage device for quantum states, into the QIP systems. For example, the concept of quantum repeater [5] has attracted much attention especially when the atomic-ensemble-based quantum memory was proposed to be integrated in the linear optical quantum network [6] 9], where the required resources increase polynomially along the communication distance. This means the QIP systems become scalable when quantum memories are integrated in [10, 11].

Along the direction of developing high-quality quantum memories, great progress has been achieved in the recent years. Various mediums have been adopted for quantum memories, e.g. atomic ensembles [12 14, single atoms [15, 16], ion-doped crystals [17, 18. Physical processes of electromagnetically induced transparency (EIT) 13, 18, Raman scattering 12, 14, and photon echo 17] are involved in the manipulation of quantum memories. Along the way for long-distance quantum communication network, till now the most advanced achievement is the realization of quantum repeater nodes [19, 20] based on cold-atomic-ensemble quantum memories. The memory performance, measured by its lifetime and retrieve efficiency, determines the complexity of a QIP system. Therefore continuously pushing the upper-limit of the lifetime and retrieve efficiency is indispensable for the future scalable QIP systems.
For the single-photon quantum memory with cold atomic ensembles, a lifetime of milliseconds was achieved in our former experiment by suppressing the dephasing with a long wavelength of the spin wave [21] and in another independent experiment by limiting the motion of the atoms with a one-dimensional optical lattice 22. The major sources of decoherence of these quantum memories are the atom loss due to thermal diffusion and gravity when the cold atoms are freely flying in the vacuum chamber [21, or the differential light shift when the cold atoms are confined with an optical trap 22. This can be understood when we look at the formula of the generated spin wave,

$$
|\psi\rangle_{c}=\frac{1}{\sqrt{N_{\text {at }}}} \sum_{j}^{N_{\mathrm{at}}} e^{i \omega_{j}^{h f} \cdot t} e^{i \Delta \boldsymbol{k} \cdot \boldsymbol{r}_{j}}\left|g \cdots s_{j} \cdots g\right\rangle .
$$

Here, $N_{\mathrm{at}}, \omega_{j}^{h f}, \Delta \boldsymbol{k}$, and $\boldsymbol{r}_{j}$ are the number of the atoms, the hyperfine split of the $j$ th atom, the momentum transfer from light to atoms, and the position of the $j$ th atom respectively. The two phases $\phi_{1}=\left(\omega_{j}^{h f} \cdot t\right)$ and $\phi_{2}=\left(\Delta \boldsymbol{k} \cdot \boldsymbol{r}_{j}\right)$ are correlated with the differential light shift and the motion of the atoms respectively and have to be maintained for keeping the phase pattern.

In order to eliminate these decoherence effects in our former experiment 21], in the present experiment we have developed a blue-detuned optical trap to confine the cold atoms and a weak light field to compensate the differential light shift in light of the idea in Ref. 23. As shown in the following text an improvement of the lifetime of the spin wave by 40 folds, from $0.67 \mathrm{~ms}$ to 28 $\mathrm{ms}$, has been obtained with the help of the compensation beam. Moreover, the transverse mode breathing of the singly-excited spin wave is observed for the first time and clearly interpreted by a Monte-Carlo simulation procedure.

Shown in Fig 1(a) the blue-detuned optical trap consists of a hollow beam and two light sheets both delivered from a Ti:Sapphire laser working at wavelength $775 \mathrm{~nm}$ 


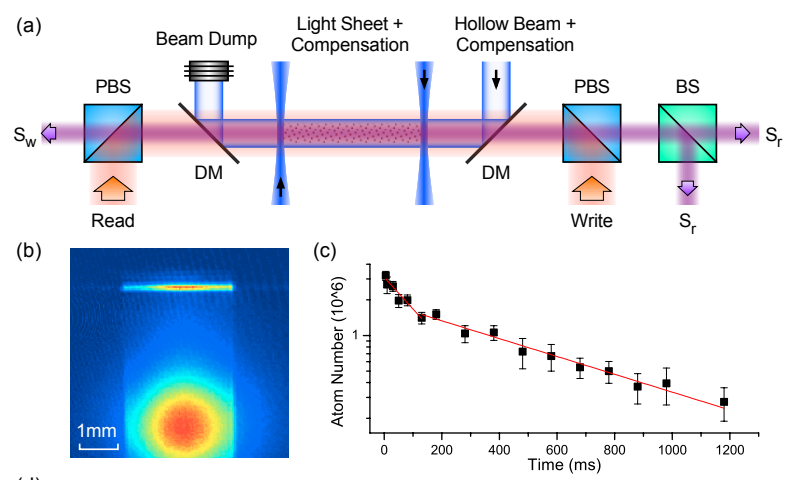

Light Sheet +
Compensation $\quad \begin{aligned} & \text { Hollow Beam }+ \\ & \text { Compensation }\end{aligned}$

(d)
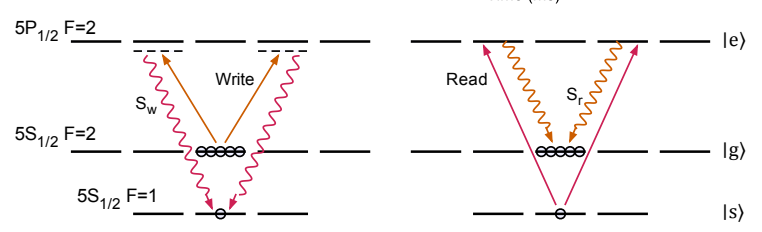

FIG. 1: A schematic of the setup. (a) The memory light modes are arranged in a collinear configuration, along the axial direction of the hollow beam. The light modes of the write, read, write-out photon $S_{w}$, and read-out photon $S_{r}$ are all focused single-mode Gaussian beams. At the focus, the diameter for the write and read is $550 \mu \mathrm{m}$ and that for the signal beams is $130 \mu \mathrm{m}$. (b) An absorption image of the atoms in the dipole trap (with false color). The dipole trap holds $\sim 3 \times 10^{6}$ atoms at $30 \mathrm{~ms}$ after sub-Doppler cooling, in a shape with length $3 \mathrm{~mm}$ and diameter $190 \mu \mathrm{m}$. (c) The loss of atoms in the optical trap. Within the first $100 \mathrm{~ms}$, the hot atoms quickly escape from the trap, and an exponential fitting gives the decay constant of $160 \mathrm{~ms}$. After that, the atom number slowly decays with a constant of $580 \mathrm{~ms}$. (d) Relevant atomic levels. A pair of clock states, $\mid F=2, m_{F}=$ $0\rangle$ and $\left|F=1, m_{F}=0\right\rangle$, are chosen as the memory states to suppress dephasing from inhomogeneous magnetic field. BS, 50:50 beam splitter; PBS, polarizing beam splitter; DM, dichroic mirror.

and a power of $1.9 \mathrm{~W}$. The hollow beam is generated by the so-called convex-axicon combination [24, which has a steep ascent of laser intensity along the radial direction. The focal length of the convex lens is $150 \mathrm{~mm}$, and the edge angle of the axicon lens is $0.073^{\circ}$. Taking a collimated Gaussian mode with a diameter of $16 \mathrm{~mm}$ as the input, the laser beam is transformed to a ring-shaped profile of $190-\mu \mathrm{m}$ inner diameter at the focus. The $3-\mathrm{mm}$ long cylindrical section around the focus is taken as the trap region. Another two light sheets with elliptical profile close the two ends of the optical trap. Unlike the reddetuned single-beam optical trap [25] where cold atoms are collected at the intensity maximum of the trapping beam, in this blue-detuned "box"-like trap cold atoms stay in the dark region. Such a trap has the advantages of lower scattering rate and smaller differential light shift over the red-detuned trap when they have the same trap depth and comparable detuning. Moreover, it is more feasible to adjust the inner diameter of the hollow beam

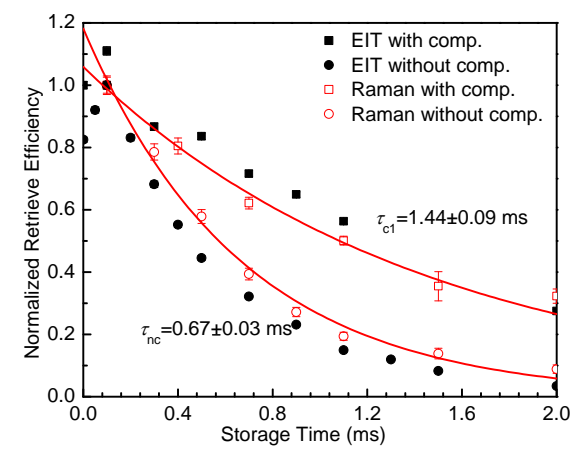

(a)



(b)
FIG. 2: (a) Retrieve efficiency at short time scale. (b) The wavelength of the trapping beam is $775 \mathrm{~nm}$ and that for that of the compensation beam is $780 \mathrm{~nm}$. Without compensation, dominated dephasing mechanism is differential light shift from the trapping laser, and an exponential fitting gives a lifetime of $0.67 \pm 0.03 \mathrm{~ms}$. When the compensation laser is turned on, the decay constant becomes $1.44 \pm 0.09 \mathrm{~ms}$.

to match the optical modes of the signal beams $S_{w}$ and $S_{r}$.

During the experiment, the dipole trap passing through the center of a dark magneto-optical trap (dark MOT) 24] is kept on all the time. Around $3 \times 10^{6}$ cold ${ }^{87} \mathrm{Rb}$ atoms at a temperature of $15 \mu \mathrm{K}$ are transferred into the dipole trap after a 150-ms dark MOT phase and a 6-ms sub-Doppler cooling phase. Then, a weak magnetic field of $340 \mathrm{mG}$ is turned on along the axial direction of the hollow beam, which defines the quantization axis. The atoms outside the dipole trap fall down and expand from the trapped atoms as shown in Fig 1 (b) by waiting for another $30 \mathrm{~ms}$. Afterwards within $100 \mu \mathrm{s}$ the trapped atoms are optically pumped to the initial state $|g\rangle$ with a measured optical depth of $9.6 \pm 1.0$ along the quantization axis. The states of $|g\rangle$ and $|s\rangle$, a pair of "clock" states, are taken as the memory states. From now, the memory cycles repeat for 10 to $300 \mathrm{~ms}$ (depending on the storage time) until a new trap loading cycle starts.

In the present experiment, both the off-resonance Raman scattering and the EIT processes are utilized to test the quality of the quantum memory. When the Raman scattering is used to generate spin excitations via $|g\rangle \rightarrow|e\rangle \rightarrow|s\rangle$, the excitation rate is controlled to about $2 \times 10^{-3}$. After a photon is detected in the signal mode $S_{w}$, it triggers the read pulse at a storage time $\Delta T$ to convert the collective excitation into a single photon in the mode of $S_{r}$. The retrieve efficiency is estimated by the ratio between the count in the $S_{r}$ channel and that in the $S_{w}$ channel. When we study the EIT process, the control beam with a power of $200 \mu \mathrm{W}$ is sent in from the read channel and the probe beam is coupled in from the $S_{w}$ channel and detected at the $S_{r}$ channel. The intensity of the probe pulse is set at single photon level. The 
memory efficiency, optimized according to an iterating procedure [26, is defined by the fraction being read out relative to the intensity of the probe pulse itself.

Since the power of the trapping laser is about $1.9 \mathrm{~W}$, its wavelength is set to $775 \mathrm{~nm}$ to maintain a trap depth of $45 \mu \mathrm{K}$. The estimated coherence time is $0.85 \mathrm{~ms}$ due to the differential light shift. A compensation laser with power of $3.5 \mu \mathrm{W}$ and frequency tuned between the two DII resonances (shown in Fig 2(b)) is mixed into the trapping laser with a beam sampler before they are coupled into a single-mode polarization-maintaining fiber. After propagating in the fiber, these two lasers share an identical profile. Taking the chromatic abberation of the following optics into account, the estimated lifetime is $\sim 300 \mathrm{~ms}$. The two lasers are frequency stabilized and the relative intensity drift between them is actively controlled to smaller than $1 \%$.

The retrieve efficiencies measured with the Raman scattering and the EIT process are shown in Fig 2(a). The detected retrieve efficiency at a storage time below $100 \mu \mathrm{s}$ is about $7 \%$ when the Raman scattering is utilized. By removing the inefficiencies of mode-matching, transmittance loss, and detection efficiency of the single photon counter module, the intrinsic retrieve efficiency is about $\eta_{\mathrm{RS}}=30 \%$. The memory efficiency with the EIT process is obtained as $\eta_{\mathrm{EIT}}=22 \%$ where the inefficiencies have been automatically removed during the measurement. These two efficiencies $\left(\eta_{\mathrm{RS}}\right.$ and $\left.\eta_{\text {EIT }}\right)$ are well comparable. In order to compare the efficiency curves, the data points are normalized to the value at short storage times. It can be seen that decay curves obtained from the two physical processes are consistent to each other. Meanwhile, in order to show the single photon nature of the retrieved photon from the spin wave generated with off-resonance Raman scattering, we measured the anticorrelation parameter [27] as $0 \pm 0.1$ at $1.2 \mathrm{~ms}$.

The fitted lifetime without the compensation beam is $0.67 \pm 0.03 \mathrm{~ms}$ which is well in agreement with the estimated value of $0.85 \mathrm{~ms}$. However, the decay constant with the compensation beam is $1.44 \pm 0.09 \mathrm{~ms}$ which is far from the estimated value of $300 \mathrm{~ms}$.

For the collinear phase-matching configuration as in Fig 1 (a), the generated spin-wave has a wavelength $\sim$ $4.4 \mathrm{~cm}$, which is much longer than the trap length 3 $\mathrm{mm}$. Therefore the dephasing from atomic thermal motion along the axial direction is suppressed and the induced decoherence is negligible. When the measurement is taken at longer storage times, obvious oscillations emerge in the curves of the memory efficiencies as in Fig 3 .

We first look at Fig 3 (a) which was measured with the Raman scattering. Two dips at $3 \mathrm{~ms}$ and $5 \mathrm{~ms}$ and two maxima at $4 \mathrm{~ms}$ and $10 \mathrm{~ms}$ are observed. The density of the atoms is about $5 \times 10^{10} \mathrm{~cm}^{-3}$, which should not induce the spin self rephasing at several milliseconds due to the identical spin rotation effect observed in a for- (a)

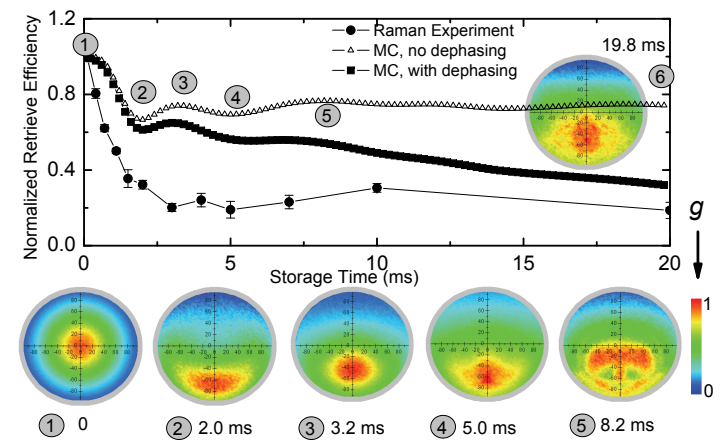

(b)

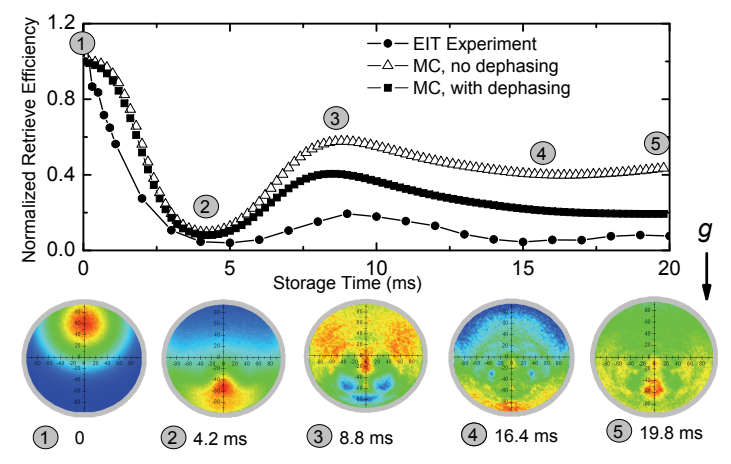

FIG. 3: Oscillation of retrieve efficiency due to dynamical mode breathing. (a) Retrieve efficiency curve when the signal mode is at the center of the trap and the spin wave is generated by off-resonance Raman scattering. (b) Retrieve efficiency curve when the signal mode is $60 \mu \mathrm{m}$ above the center of the trap and the spin wave is generated by the EIT process. The transverse spatial mode of the singly-excited spin wave at different storage times is simulated with a Monte-Carlo procedure and shown below the retrieve efficiency curves. The triangle points are the overlapping integral calculated directly from the initial spatial mode and the evolved spatial mode. The squared dots are obtained by considering atom loss and the dephasing time obtained in Fig 4 The color depth shows the density of the atoms and is re-scaled for each figure to make the distribution more visible.

mer experiment 28. In order to explore the mechanism for the phase revival, we construct a model for numerically simulating motions of the atoms in the trap with a Monte-Carlo procedure.

When a single photon is registered in the $S_{w}$ channel which has a Gaussian profile defined by the single-mode fiber and its collimator, the spin wave possesses a Gaussian profile accordingly with a initial transverse distribution of $U(x, y, t=0)$. After a period of thermal expansion and free falling, the transverse spatial mode of the spin wave evolves into $U(x, y, t=\Delta T)$. Then we define the retrieve efficiency as the overlap of the two modes, i.e. $R(\Delta T)=\left|\iint \mathrm{d} x \mathrm{~d} y \sqrt{U(x, y, 0)} \sqrt{U(x, y, \Delta T)}\right|^{2}$. As the two spatial modes $U(x, y, 0)$ and $U(x, y, \Delta T)$ are identical at $\Delta T=0$, their overlap is unit. At $\Delta T=2.0 \mathrm{~ms}$, the atoms get more diffused and fall down to the lower part of the trap. Then a dip appears in the efficiency curve due to the decreasing overlap. At $\Delta T=3.2 \mathrm{~ms}$, 


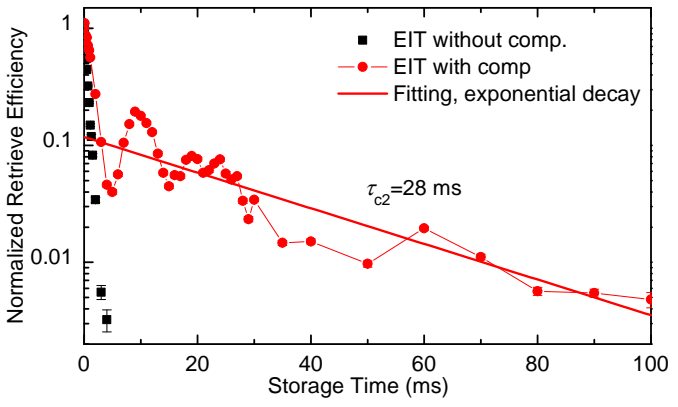

FIG. 4: Memory efficiency at long time scale. By a leastsquare fitting with an exponential function to the measured data, a decay constant of $28 \pm 2.5 \mathrm{~ms}$ is obtained.

the atoms are bounced higher and more concentrated. Therefore the overlap becomes larger and a maximum arises. At $\Delta T=5.0 \mathrm{~ms}$, the atoms get diffused again and the overlap becomes smaller therefore the second dip appears. Until $\Delta T=8.2 \mathrm{~ms}$, even though the atoms expand more which should reduce the overlap, the position of the atoms is closer to the initial position which should increase the overlap. As a result of the competition between these two effects, a flat maximum arises. After a sufficiently long time until $19.8 \mathrm{~ms}$, the distribution of the atoms becomes more chaotic and the oscillations disappears. The features in the experimental curve are reproduced in the Monte-Carlo simulation according to the transverse mode breathing although the positions are slightly shifted. This discrepancy may come from the deviation of the trap diameter from $190 \mu \mathrm{m}$, the inhomogeneity of the trap and the imperfection of the simulation model.

In order to make this dynamical mode breathing effect more visible, we shifted the signal beam $60 \mu \mathrm{m}$ above the trap center. As shown in Fig 3 (b), the oscillations of memory efficiency become much more evident. The features of the revivals in the experimental result are all reproduced in the Monte-Carlo simulations. As explained above, the discrepancies between the experimental curve and the simulated results should arise from the similar reasons.

At even longer time scale, the memory efficiency curve is measured up to $100 \mathrm{~ms}$. By a least-square fitting to the experimental data with an exponential function, a lifetime of $28 \pm 2.5 \mathrm{~ms}$ is obtained, which indicates an improvement by 40 folds. However, it is still much shorter than the estimated value of $300 \mathrm{~ms}$. This is due to the spatial mode mismatching between the trap beam and the compensation beam arising from the imperfection of the optics. Moreover, the relative intensity stability of the compensation beam of $1 \%$ limits the compensation effect as well.

In summary, the present work showed a 40-fold improvement of the coherence time of the quantum memory based on optically trapped atoms with a compensation laser beam. Especially, the transverse mode breathing effect, a collective behavior of the non-interactive atoms, of a singly-excited spin wave was observed and elucidated with a Monte-Carlo simulation procedure. Since this effect is induced by the transverse mode diffusion and revival instead of the evolution of the phase pattern of the spin wave, it is not likely to be observed with the traditional Ramsey spectroscopy. The present experiment suggests that a three dimensional optical lattice is necessary for completely suppressing the spatial mode diffusion of atoms. With detailed analysis of the decoherence mechanisms, this work provides a benchmark for the further development of high-quality quantum memories.

This work was supported by the European Commission through the ERC Grant and the STREP project HIP, the CAS, the NNSFC and the National Fundamental Research Program (Grant No. 2011CB921300) of China.

Note added: During the course of our experiment, we learnt a parallel effort for extending the lifetime of a quantum memory by about one order with the technique of compensating the differential light shift in a reddetuned one-dimensional optical lattice [29].

[1] M. A. Nielsen and I. L. Chuang. Quantum Computation and Quantum Information. Cambridge University Press (2000).

[2] E. Knill, et al. Nature 409, 46 (2001).

[3] N. Gisin, et al. Rev. Mod. Phys. 74, 145 (2002).

[4] J.-W. Pan, et al. arXiv:0805.2853 (2008).

[5] H.-J. Briegel, et al. Phys. Rev. Lett. 81, 5932 (1998).

[6] L.-M. Duan, et al. Nature 414, 413 (2001).

[7] B. Zhao, et al. Phys. Rev. Lett. 98, 240502 (2007).

[8] L. Jiang, et al. Phys. Rev. A 76, 012301 (2007).

[9] O. A. Collins, et al. Phys. Rev. Lett. 98, 060502 (2007).

[10] H. J. Kimble. Nature 453, 1023 (2008).

[11] Z.-S. Yuan, et al. Physics Reports 497, 1 (2010).

[12] D. N. Matsukevich and A. Kuzmich. Science 306, 663 (2004).

[13] M. D. Eisaman, et al. Nature 438, 837 (2005).

[14] Y.-A. Chen, et al. Nat. Phys. 4, 103 (2008).

[15] D. L. Moehring, et al. Nature 449, 68 (2007).

[16] W. Rosenfeld, et al. Phys. Rev. Lett. 98, 050504 (2007).

[17] H. de Riedmatten, et al. Nature 456, 773 (2008).

[18] J. J. Longdell, et al. Phys. Rev. Lett. 95, 063601 (2005).

[19] C.-W. Chou, et al. Science 316, 1316 (2007).

[20] Z.-S. Yuan, et al. Nature 454, 1098 (2008).

[21] B. Zhao, et al. Nat. Phys. 5, 95 (2009).

[22] R. Zhao, et al. Nat. Phys. 5, 100 (2009).

[23] A. Kaplan, et al. J. Opt. B-Quantum Semicl. Opt. 7, R103 (2005).

[24] F. Yang, et al. (in preparation).

[25] C.-S. Chuu, et al. Phys. Rev. Lett. 101, 120501 (2008).

[26] I. Novikova, et al. Phys. Rev. Lett. 98, 243602 (2007).

[27] S. Chen, et al. Phys. Rev. Lett. 97, 173004 (2006).

[28] C. Deutsch, et al. Phys. Rev. Lett. 105, 020401 (2010).

[29] A. G. Radnaev, et al. Nat Phys 6, 894 (2010). 